\title{
Mongolian Domestic Tourists' Motivation and Revisit Intention: Mediating Effect of Perceived Benefit and Perceived Value
}

\author{
Oyunchimeg Luvsandavaajav ${ }^{1}$ iD, Gantuya Narantuya ${ }^{2}$ \\ ${ }^{1}$ Department of Geography, National University of Mongolia; Ikh Surguuliin Street 1, Ulaanbaatar 14201, Mongolia; \\ Faculty of Business and Economics, University of Pecs, H-7622 Pécs, Rákóczi út 80, Hungary \\ 2Department of Geography, National University of Mongolia; Ikh Surguuliin Street 1, Ulaanbaatar 14201, Mongolia \\ oyunchimeg_l@num.edu.mn (O.L.); n.gantuya@num.edu.mn (G.N.)
}

Received: 25 March 2021; Revised: 11 May 2021; Accepted: 13 May 2021; Published online: 25 May 2021

\begin{abstract}
An understanding of domestic tourists' motivation and its relationship between perceived value and revisit intention is important for prediction of future travel demand. This study aims to examine domestic tourists' motivation on revisit intention mediated by perceived benefits and perceived values. The quantitative data was collected through self-administered questionnaires from domestic tourists who travelled in Mongolia in the summer of 2019. An effect of domestic tourists' travel motivation on revisit intentions; and the mediating effect of perceived benefit and perceived value were analyzed through factor analysis, regression analysis to test the proposed research hypotheses. The research result indicates that the mean ranking of the constructs of travel motivations for Mongolian domestic tourists were escape from daily life, relaxation, sightseeing, affordability of services, seeking variety, and culture were the top reasons to travel to the local destinations. The research findings contribute to the notion of domestic tourists' travel motivation, its relationship between perceived benefit, perceived value of their trip on their willingness to return and recommendations to others. Furthermore, the research result could be beneficial for destination planners and tourism practitioners to enhance destination attributes and launch a variety of products, services, affordable pricing and accessible environment. Overall, the result provides background for an appropriate and effective marketing strategy to develop domestic tourism in Mongolia; and increase the competitiveness of the destinations with higher length of stay and more tourist spending.
\end{abstract}

Key words: Mongolia, domestic tourists, push and pull travel motivations, mediating factors, revisit intention

Citation: Oyunchimeg, L., \& Gantuya, N. (2021). Mongolian Domestic Tourists' Motivation and Revisit Intention: Mediating Effect of Perceived Benefit and Perceived Value. Central European Journal of Geography and Sustainable Development, 3(1), 32-48. https://doi.org/10.47246/CEJGSD.2021.3.1.3 